\section{RMD Open}

Rheumatic \&

Musculoskeletal Diseases

\title{
Factors influencing the use of
} tocilizumab as monotherapy in patients with rheumatoid arthritis in a real-life setting: results at 1 year of the ACT-SOLO study

To cite: Flipo R-M, Maillefert J-F, Chazerain $P$, et al. Factors influencing the use of tocilizumab as monotherapy in patients with rheumatoid arthritis in a reallife setting: results at 1 year of the ACT-SOLO study. RMD Open 2017;3:e000340. doi:10.1136/rmdopen-2016000340

- Prepublication history and additional material is available. To view please visit the journal (http://dx.doi.org/ 10.1136/rmdopen-2016000340).

Received 19 July 2016 Revised 22 November 2016 Accepted 8 December 2016

CrossMark

For numbered affiliations see end of article.

Correspondence to Dr René-Marc Flipo; Rene-Marc.FLIP0@chru-lille.fr

\section{ABSTRACT}

Introduction: Using a biologic disease-modifying antirheumatic drug (bDMARD) as monotherapy in clinical practice for patients with rheumatoid arthritis $(\mathrm{RA})$ is common and recognised by health authorities although current guidelines recommend to combine them with conventional synthetic (cs)DMARDs. This study mainly aimed to search for real-life factors influencing the use of tocilizumab as MONO or in combination (COMBO).

Methods: In this non-interventional, prospective, national, multicentre study, data were collected every 3 months over a 12-month period in RA patients starting tocilizumab. The proportion of monotherapy patients was described, together with significant explicative factors.

Results: Among the 577 analysed patients recruited from January 2012 to August 2013 (228 monotherapy patients; 40\%), 79\% were women, mean RA duration was $11 \pm 9$ years, previous RA treatments included bDMARDs and csDMARDs in $75 \%$ of cases and mean Disease Activity Score 28 joints-Erythrocyte Sedimentation Rate (DAS28-ESR) was $5.2 \pm 1.3$ at inclusion. Explicative factors for monotherapy were at least 65 years $(O R=1.47, p=0.0485)$, no methotrexate within the two last years $(0 R=5.96, p<0.0001)$, past severe infection $(O R=1.99, p=0.0272)$ and higher baseline DAS28-ESR (OR=1.22, $\mathrm{p}=0.0086)$. Regarding clinical results (DAS28-ESR, Clinical Disease Activity Index (CDAI) and Simple Disease Activity Index (SDAI) low disease activity and remission; ACR20/50/70 and European League Against Rheumatism (EULAR) response; Health Assessment Questionnaire Disability Index (HAQ-DI) score), no relevant differences between monotherapy and combination patients were observed at 1 year. A total of 23 tocilizumab-treated patients (4\%) experienced serious infections; no new safety signals were noted with no differences between groups.

Conclusions: ACT-SOLO confirms the high proportion of RA patients receiving tocilizumab as MONO in clinical practice. The study also showed that clinical results at

\section{Key messages}

What is already known about this subject? Using a biologic disease-modifying antirheumatic drug (bDMARD) as monotherapy in patients with rheumatoid arthritis (RA) in clinical practice is common and recognised by health authorities although current guidelines recommend to combine them with conventional synthetic (cs)DMARDs for better efficacy.

What does this study add?

- MONO with tocilizumab was associated with no treatment with methotrexate within the past 2 years, elderly patients (at least 65 years old), past history of severe infection and more active disease.

- At 1 year after the first tocilizumab infusion, clinical results were similar between patients receiving the drug as monotherapy or in combination with a conventional synthetic DMARD which is consistent with RCT results.

How might this impact on clinical practice?

- Tocilizumab is used as MONO in older comorbid patients with RA with satisfactory clinical efficacy and tolerance.

1 year were similar between MONO and COMBO patients in a real-life setting.

Trial registration number: NCT01474291.

\section{INTRODUCTION}

Rheumatoid arthritis (RA) is the most common of the chronic inflammatory rheumatic diseases, affecting $0.3-1 \%$ of the population, characterised by joint pain and 
swelling, progressive functional disability due to persistent synovitis and increased morbidity and mortality. ${ }^{1-4}$ The management of RA had taken an important step with the development of new biologic disease-modifying antirheumatic drugs (bDMARDs). Among them, tocilizumab, a humanised, monoclonal antibody against interleukin-6 soluble and membrane-bound receptors, has shown its efficacy and safety in combination with methotrexate (MTX) for the treatment of moderate to severe active RA, ${ }^{5-9}$ and as monotherapy. ${ }^{8} 1011$

International and national guidelines recommend to use bDMARDs in combination with conventional synthetic (cs)DMARDs ${ }^{12-15}$ for better efficacy, and recognise the use of biologics as monotherapy when the combination with a csDMARD is not possible. Monotherapy is common in clinical practice as it concerns around $30 \%$ of patients. ${ }^{16-19}$

In this context, the ACT-SOLO study primarily aimed to search for real-life factors influencing the use of tocilizumab as MONO or in combination with a csDMARD in RA patients. Secondary and explorative objectives included the description of patients and disease characteristics, reasons leading to tocilizumab therapeutic strategy, efficacy and tolerability of tocilizumab, evolution of patients' quality of life under treatment, predictive factors of maintenance of tocilizumab treatment until 1 year (M12), glucocorticoids and csDMARDs over study period and RA activity at M12.

\section{METHODS}

This French non-interventional (ie, observational), multicentre and prospective cohort study was managed in cooperation with an independent scientific committee including three hospital-based rheumatologists.

In accordance with French law regarding noninterventional studies, ACT-SOLO protocol (NCT01474291) was approved by the Comité Consultatif sur le Traitement de l'Information en Matière de Recherche dans le Domaine de la Santé (Consultative Committee on Information Processing for Research in the Field of Health) and was validated by the Commission Nationale de l'Informatique et des Libertés (Independent Administrative Authority Protecting Privacy and Personal Data), which guarantee confidentiality to the subjects. All patients were informed about the course of the study before enrolment.

\section{Participating physicians and patients}

From the 1132 French rheumatologists regularly managing RA patients who were invited to participate in the study, $161(14 \%)$ physicians with active annual file of at least $20 \mathrm{RA}$ agreed and 118 (10\%) investigators included at least one eligible patient in ACT-SOLO study from January 2012 to August 2013. The last patient last visit was performed on September 2014.

Eligible patients for the study were adults starting tocilizumab treatment for RA either in combination with a
csDMARD (COMBO) or as MONO, and who agreed to participate; patients participating in a clinical trial on RA at the time of inclusion were excluded.

\section{Data collected}

Main characteristics of participant rheumatologists were collected (age, gender, type of practice, main medical facility and geographic location).

At the inclusion visit (at tocilizumab start), investigators reported patients and RA characteristics, prior and concomitant RA treatments, efficacy indexes (Disease Activity Score 28 joints-Erythrocyte Sedimentation Rate (DAS28), Clinical Disease Activity Index (CDAI), Simple Disease Activity Index (SDAI), American College of Rheumatology (ACR) 20/50/70, and European League Against Rheumatism (EULAR) and American College of Rheumatology (ACR) 20/50/70 responses), patient's and physician's Visual Analogue Scale (VAS) on global assessment of disease activity and use of tocilizumab. At the follow-up visits (around M3, M6 and M12 after inclusion), following additional patient data were collected: changes in RA therapy (with reasons) and adverse events (AEs, serious AEs (SAEs), and AEs of special interest for tocilizumab (AESIs: anaphylaxis/hypersensitivity reactions, demyelinating disorders, gastrointestinal perforations, malignancies, myocardial infarctions/acute coronary syndromes, strokes, and serious and/or medically significant infections, hepatic events and bleeding events)). At each visit, patients fulfilled a self-reported questionnaire (VASs on pain, fatigue and global assessment of disease activity) completed with functional and quality of life questionnaires (Health Assessment Questionnaire Disability Index (HAQ-DI) ${ }^{20}$ Rheumatoid arthritis Impact of Disease scale (RAID) ${ }^{21}$ and Patient Acceptable Symptom State (PASS) except at M3.

\section{Study size and statistical methods}

On the basis of findings from recent French studies conducted in current medical practice, ${ }^{22}{ }^{23}$ the proportion of RA patients receiving tocilizumab as MONO was estimated between $30 \%$ and $40 \%$. In order to meet study primary objective (ie, to describe the factors influencing the use of tocilizumab as MONO or in combination), sample size was computed to assess an OR of 2.00 at $5 \%$ significance with a power of at least $90 \%$, for a broad range of exposure levels (20-50\%) and a proportion of patients with tocilizumab as MONO of $30-40 \%$. Among those various calculations, maximum sample size required was 589 patients.

Statistical analyses were performed using SAS software (V.9.2). All tests were two-sided with $\alpha$ risk at 5\%.

Efficacy analyses were performed on the population of included patients who met all the selection criteria and received at least one tocilizumab infusion (efficacy population). Two groups of patients were defined according to the use of tocilizumab, as MONO or in combination with a csDMARD at treatment initiation (COMBO). Safety data were analysed on the population of patients 
with at least one tocilizumab infusion (safety population).

Regarding the primary criterion of the study, explicative factors of the tocilizumab strategy (MONO or COMBO) were looked for among baseline patients and RA characteristics, previous RA treatments and physicians' characteristics (ie, sociodemographic, type and year of practice start and geographic location), using generalised estimating equation logistic regression so as to take into account the correlation of patients in the same centre. Univariate analyses were used to select $(p \leq 0.10)$ the explanatory variables to include in the multivariate model, then statistically significant $(\mathrm{p} \leq 0.05)$ or medically relevant parameters were retained for the multivariate model. Second, multiple imputation method was used to replace missing data.

The rate of the maintenance of tocilizumab treatment (proportion of patients still treated with at M12, addition or change in csDMARD not taken into account) until M12 was described, and analyses were carried out using the Kaplan-Meier method to estimate the median maintenance duration and rate until M12.

The secondary efficacy endpoints were described at each follow-up time point as well as changes from baseline. The proportions of patients with ACR 20/50/70 and EULAR response (good or moderate) were also described as well as the proportions of patients in remission and low disease activity (LDA) in terms of DAS28-ESR $(<2.6$ and $\leq 3.2$, respectively), CDAI $(\leq 2.8$ and $\leq 10)$ and SDAI $(\leq 3.3$ and $\leq 11)$. These analyses were performed in all the patients (missing data considered as failure) and in completed patients (excluding missing data).

A propensity score (probability of receiving tocilizumab as MONO rather than in combination) was built in order to control confounding covariates for comparison of efficacy between monotherapy and combination. It was estimated using a multivariate logistic regression model (stepwise selection, entry and retain thresholds: 0.30 for both). Tested parameters taken into account for analysis included baseline patients' characteristics, baseline RA characteristics and activity, medical history and comorbidities of patients, previous RA treatments (type of treatments and MTX), dosage of glucocorticoids at tocilizumab initiation and baseline RAID score. Comparison of drug retention rate, steroid use and RA activity between monotherapy and combination were adjusted on propensity score using inverse probability weighting.

After univariate analyses used to preselect significant $(p \leq 0.30)$ or medically relevant parameters, a multivariate model was built (stepwise selection, entry and retain thresholds: 0.15 and 0.10 , respectively) to search for predictive factors of maintenance of tocilizumab treatment until M12, glucocorticoids prescription at M12 and RA activity at M12. Tested parameters were the same as those used for the calculation of the propensity score.
RESULTS

Participating physicians and patients

By comparison with physicians included in the national selection database compiling all French rheumatologists regularly managing RA patients (1132 eligible specialists), the characteristics of the 118 investigators participating in the ACT-SOLO study were comparable in terms of geographical location. Considering that tocilizumab has to be prescribed at hospital first, more participant physicians practiced in hospitals only compared with the physicians from the source population.

Among the 608 included patients, 577 patients fulfilled all the selection criteria and were analysed in the efficacy population (228 patients $(40 \%)$ in the MONO group and $349(60 \%)$ within the COMBO group) (figure 1). The proportion of monotherapy patients was consistent with the hypothesis of the sample size calculation of the study protocol.

Five patients (no tocilizumab infusion $(n=4)$, duplicate patient $(n=1))$ were excluded from the safety population $(n=603)$.

\section{Patients' baseline characteristics}

At inclusion, RA and patients' characteristics were generally numerically less favourable in the MONO group (table 1).

RA monotherapy patients were slightly older than combination patients ( $59 \pm 13$ vs $55 \pm 13$ years) and they presented with past diseases or comorbidities more often $(76 \%$ vs $68 \%$ of patients; arterial hypertension: $28 \%$ vs $21 \%$, dyslipidaemia: $23 \%$ vs $14 \%$, past lung disease: $20 \%$ vs $14 \%$ and osteoporosis: $18 \%$ vs $14 \%$ ). RA duration was longer in the MONO group when compared with the COMBO group with $45 \%$ and $41 \%$ of patients presented with RA for more than 10 years.

As shown in table $1,94.6 \%$ of all the RA patients received MTX at least once before tocilizumab initiation. Within the two last years, among the 543 patients having received MTX at least once, only $52 \%$ of monotherapy patients were treated with MTX while combination patients were $89 \%$ in this case. Most of the patients $(76 \%)$ received at least one previous or ongoing bDMARD and $66 \%$ received oral glucocorticoids (mean dose $10 \pm 7 \mathrm{mg} /$ day eq. prednisone) at the time of inclusion, without relevant differences between patients' groups. At baseline, monotherapy patients presented with higher disease activity than combination patients (DAS28-ESR: $5.4 \pm 1.3$ vs 5.0 \pm 1.2 ; CDAI: $29.3 \pm 13.7$ vs 25.7 \pm 11.4 ; SDAI: $31.9 \pm 14.9$ vs $27.2 \pm 12.1$; HAQ-DI $1.66 \pm 0.63$ vs $1.49 \pm 0.64$; worsening of structural damage over the past 2 years: $51.4 \%$ vs $46.6 \%$ ) (table 1 ).

\section{Explicative factors of the tocilizumab strategy (MONO or COMBO) \\ Univariate analyses}

Univariate analyses conducted on observed data showed nine significant and medically relevant predictor parameters for the initiation of tocilizumab as MONO: 
Figure 1 Study flow chart. COMBO, tocilizumab in combination with csDMARD at inclusion; eCRF, electronic Case Report Form; MONO, tocilizumab as monotherapy at inclusion; RA, rheumatoid arthritis. *Two patients presented with two exclusion criteria: patient previously treated with tocilizumab and participation in a clinical trial at inclusion $(n=1)$, patient with no RA and no treatment with tocilizumab $(n=1)$.

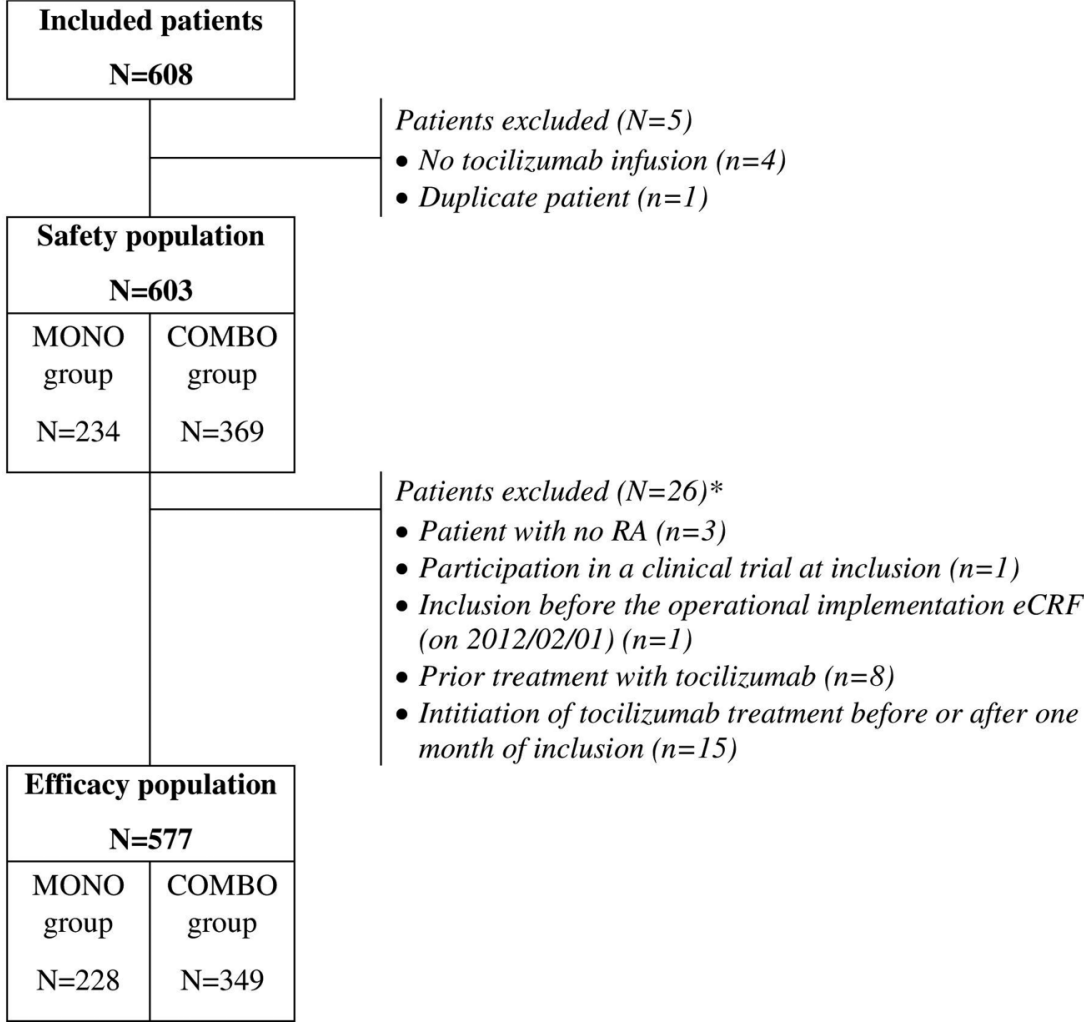

patient's age $( \pm 65$ years: $\mathrm{p}=0.0026), \quad \mathrm{RA}$ duration ( \pm 15 years: $p=0.0422)$, MTX within the two last years $(\mathrm{p}<0.0001)$, arterial hypertension $(\mathrm{p}=0.0560)$, pulmonary disease $(\mathrm{p}=0.0163)$, gastrointestinal disease $(\mathrm{p}=0.0626)$, past infectious disease $(\mathrm{p}=0.0817)$, dyslipidaemia $(\mathrm{p}=0.0018)$, mean CRP or mean DAS28-ESR values $(\mathrm{p}=0.0024$ and $\mathrm{p}=0.0018)$.

\section{Multivariates analyses}

Five of the nine retained parameters from univariate analyses were found as independent explicative factors of tocilizumab prescription as MONO, using multivariate analysis: dyslipidaemia $(\mathrm{OR}=2.04,95 \%$ CI 1.33 to 3.14 , $\mathrm{p}=0.0011)$, at least 65 years of age $(\mathrm{OR}=1.47,95 \% \mathrm{CI}$ 1.00 to $2.15, \mathrm{p}=0.0485)$, no MTX use within the two last years $(\mathrm{OR}=5.96,95 \%$ CI 4.08 to $8.70, \mathrm{p}<0.0001)$, past history of severe infectious disease $(\mathrm{OR}=1.99,95 \% \mathrm{CI}$ 1.08 to 3.66, $\mathrm{p}=0.0272$ ) and higher baseline DAS28-ESR (unit=1, OR=1.22, 95\% CI 1.05 to $1.42, \mathrm{p}=0.0086$ ). Dyslipidaemia was finally excluded to the second multivariable analysis because this parameter was strongly correlated with age and led to hamper epidemiological informations in multivariate analysis (coefficient of variation between models with and without dyslipidaemia: $6 \%$, ie, $<10 \%)$. These reasons led to perform second multivariate analysis excluding this parameter. Then, multivariate analysis showed the same four independent explicative factors of tocilizumab prescription as MONO: at least 65 years of age $(\mathrm{OR}=1.56,95 \%$ CI 1.06 to 2.30, $\mathrm{p}=0.0230)$, no MTX use within the last two years $(\mathrm{OR}=5.74,95 \%$ CI 3.92 to $8.43, \mathrm{p}<0.0001)$, past history of severe infectious disease $(\mathrm{OR}=2.03,95 \%$ CI 1.11 to $3.70, \mathrm{p}=0.0212$ ) and higher baseline DAS28-ESR (unit=1, $\mathrm{OR}=1.22,95 \%$ CI 1.05 to $1.41, \mathrm{p}=0.0076$ ) (figure 2 ).

\section{Use of tocilizumab}

Mean starting dose of tocilizumab was $7.9 \pm 0.5 \mathrm{mg} / \mathrm{kg}$ and duration of the first treatment infusion was 62 $\pm 12 \mathrm{~min}$. Over the study period, the mean number of tocilizumab infusions was $9 \pm 4$ and $49 \%$ of patients received at least 12 infusions of treatment. Mean time between two tocilizumab infusions was $32 \pm 13$ days without differences between patients' groups. Over the treatment period, MONO patients with at least one temporary discontinuation were less numerous $(25 \%$ vs $33 \%$ ) (table 2).

\section{Efficacy}

Using the Kaplan-Meier method, the median rate of retention in tocilizumab treatment until M12 (proportion of patients still treated with tocilizumab at M12 without csDMARD changes imputation) was 69\% $(95 \%$ CI $65 \%$ to $73 \%$ ) without difference between patients' groups (MONO: $67 \%, 95 \%$ CI $60 \%$ to $73 \%$; COMBO: $71 \%, 95 \%$ CI $65 \%$ to $76 \%$ ). Using the propensity score for adjustment on RA and patients characteristics, no statistical difference between tocilizumab as MONO and in combination with a csDMARD was observed on treatment retention until $\mathrm{M} 12(\mathrm{HR}=1.19 ; 95 \%$ CI 0.85 to 1.67; $\mathrm{p}=0.303)$. After univariate analyses, multivariate analysis showed that female patients $(\mathrm{p}=0.007)$, patients with erosive RA ( $p=0.043)$ and patients with past lung or 
Table 1 Patient and disease characteristics at baseline-efficacy population $(\mathrm{N}=407)$

\begin{tabular}{|c|c|c|c|}
\hline & $\begin{array}{l}\text { MONO group } \\
\mathrm{N}=228\end{array}$ & $\begin{array}{l}\text { COMBO group } \\
\mathrm{N}=349\end{array}$ & $\begin{array}{l}\text { All patients } \\
\mathrm{N}=577\end{array}$ \\
\hline \multicolumn{4}{|l|}{ Patients' characteristics } \\
\hline Age (years) ${ }^{\star}$ & $59.1 \pm 12.7$ & $55.3 \pm 12.5$ & $56.8 \pm 12.7$ \\
\hline Women, n (\%) & $180(78.9)$ & $274(78.5)$ & $454(78.7)$ \\
\hline \multirow[t]{2}{*}{ BMI $\left(\mathrm{kg} / \mathrm{m}^{2}\right)$} & $(n=217)$ & $(n=338)$ & $(n=555)$ \\
\hline & $25.7 \pm 5.5$ & $25.8 \pm 5.8$ & $25.8 \pm 5.6$ \\
\hline Patients with $\geq 1$ comorbidity, $n$ (\%) & $173(75.9)$ & $236(67.6)$ & 409 (70.9) \\
\hline \multicolumn{4}{|l|}{ RA history } \\
\hline RA duration (years) & $12.1 \pm 10.2$ & $10.2 \pm 8.2$ & $10.9 \pm 9.1$ \\
\hline \multirow[t]{2}{*}{ Positive RF or ACPA, n (\%) } & $(n=223)$ & $(n=336)$ & $(n=559)$ \\
\hline & $197(88.3)$ & $281(83.6)$ & $478(85.5)$ \\
\hline \multirow[t]{2}{*}{ Erosive RA, n (\%) } & $(n=225)$ & $(n=340)$ & $(n=565)$ \\
\hline & $174(77.3)$ & $261(76.8)$ & $435(77.0)$ \\
\hline Structural damage & $(n=210)$ & $(\mathrm{n}=307)$ & $(n=517)$ \\
\hline Worseningt, n (\%) & $108(51.4)$ & $143(46.6)$ & $251(48.5)$ \\
\hline \multicolumn{4}{|l|}{ Previous/ongoing treatment of RA } \\
\hline Previous/current DMARDs & $(n=225)$ & $(n=349)$ & $(n=574)$ \\
\hline Naive, $\mathrm{n}(\%)$ & $2(0.9)$ & 0 & $2(0.3)$ \\
\hline Only csDMARDs, n (\%) & $47(20.9)$ & $91(26.1)$ & $138(24.0)$ \\
\hline Only biologics, $\mathrm{n}(\%)$ & $4(1.8)$ & 0 & $4(0.7)$ \\
\hline Biologics + csDMARDs, n (\%) & $172(76.4)$ & $258(73.9)$ & $430(74.9)$ \\
\hline \multicolumn{4}{|l|}{ csDMARDs } \\
\hline \multirow{2}{*}{ Number of different previous or ongoing csDMARDs } & $(n=219)$ & $(n=349)$ & $(n=568)$ \\
\hline & $1.7 \pm 0.6$ & $1.5 \pm 0.6$ & $1.6 \pm 0.6$ \\
\hline \multirow[t]{2}{*}{ Previous/ongoing MTX, n (\%) } & $(n=225)$ & $(n=349)$ & $(n=574)$ \\
\hline & $210(93.3)$ & $333(95.4)$ & $543(94.6)$ \\
\hline Main reasons for MTX discontinuation, $\mathrm{n}(\%)$ & $(n=202)$ & $(n=55)$ & $(n=257)$ \\
\hline Intolerance & $146(72.3)$ & $45(81.8)$ & $191(74.3)$ \\
\hline Therapeutic escape & $26(12.9)$ & $4(7.3)$ & $30(11.7)$ \\
\hline \multicolumn{4}{|l|}{ bDMARDs } \\
\hline \multirow[t]{2}{*}{ Number of previous biologics } & $(n=176)$ & $(n=258)$ & $(n=434)$ \\
\hline & $1.9 \pm 1.0$ & $1.9 \pm 1.0$ & $1.9 \pm 1.0$ \\
\hline Anti-TNF agentł, $\mathrm{n}(\%)$ & $171(97.2)$ & $238(92.2)$ & $409(94.2)$ \\
\hline Oral glucocorticoids at baseline, $\mathrm{n}(\%)$ & $(n=227)$ & $(n=349)$ & $(n=576)$ \\
\hline Yes, n (\%) & $152(67.0)$ & $229(65.6)$ & $381(66.1)$ \\
\hline Daily dose (mg equivalent prednisone), $\mathrm{n}(\%)$ & $(n=226)$ & $(n=348)$ & $(n=574)$ \\
\hline 0 & $75(33.2)$ & $120(34.5)$ & $195(34.0)$ \\
\hline$[0-5]$ & $44(19.5)$ & $65(18.7)$ & $109(19.0)$ \\
\hline$[5-7.5]$ & $16(7.1)$ & $47(13.5)$ & $63(11.0)$ \\
\hline$[7.5-10]$ & $49(21.7)$ & $71(20.4)$ & $120(20.9)$ \\
\hline$>10$ & $42(18.6)$ & 45 (12.9) & $87(15.2)$ \\
\hline \multicolumn{4}{|l|}{ RA characteristics at inclusion } \\
\hline \multirow[t]{2}{*}{ HAQ-DI standard score } & $(n=167)$ & $(n=247)$ & $(n=414)$ \\
\hline & $1.66 \pm 0.63$ & $1.49 \pm 0.64$ & $1.56 \pm 0.64$ \\
\hline \multirow[t]{2}{*}{ DAS28-ESR } & $(n=220)$ & $(n=335)$ & $(n=555)$ \\
\hline & $5.38 \pm 1.32$ & $5.03 \pm 1.20$ & $5.17 \pm 1.26$ \\
\hline \multirow[t]{2}{*}{ CDAI } & $(n=205)$ & $(n=314)$ & $(n=519)$ \\
\hline & $29.29 \pm 13.72$ & $25.69 \pm 11.38$ & $27.11 \pm 12.47$ \\
\hline \multirow[t]{2}{*}{ SDAI } & $(n=200)$ & $(n=303)$ & $(n=503)$ \\
\hline & $31.92 \pm 14.93$ & $27.21 \pm 12.05$ & $29.08 \pm 13.45$ \\
\hline \multicolumn{4}{|l|}{ Patients' quality of life } \\
\hline \multirow[t]{2}{*}{ RAID Score } & $(n=170)$ & $(n=252)$ & $(n=422)$ \\
\hline & $6.5 \pm 2.0$ & $5.9 \pm 1.9$ & $6.1 \pm 1.9$ \\
\hline \multirow[t]{2}{*}{ PASS questionnaire-acceptable state, n (\%) } & $(n=168)$ & $(n=246)$ & $(n=414)$ \\
\hline & $43(25.6)$ & $84(34.1)$ & $127(30.7)$ \\
\hline
\end{tabular}

${ }^{*}$ Data are presented as mean \pm SD unless stated otherwise.

†Worsening of joint structural damage at X-ray over the past 2 years.

$\ddagger$ Adalimumab or infliximab or etanercept or certolizumab or golimumab.

ACPA, anti-citrullinated protein antibody; BMI, body mass index; CDAI, clinical disease activity index; cs/bDMARD, conventional synthetic/ biologic disease-modifying antirheumatic drug; COMBO, tocilizumab in combination with csDMARD at inclusion; DAS28-ESR, Disease Activity Score 28 joints-Erythrocyte Sedimentation Rate; ESR, Erythrocyte Sedimentation Rate; HAQ-DI, health assessment questionnaire-disease index; MONO, tocilizumab as monotherapy at inclusion; PASS, patient acceptable symptom state; RA, rheumatoid arthritis; RAID, rheumatoid arthritis impact of disease; RF, rheumatoid factor; SDAI, simple disease activity index; SJC, swollen joint count; TNF, tumour necrosis factor. 
infectious diseases $(p=0.003)$ were associated to a statistically significant increased risk of non-maintenance of treatment with tocilizumab until M12. In contrast, dyslipidaemia was associated to a statistically significant increased chance of treatment maintenance $(p=0.048)$ (data provided in online supplementary material).

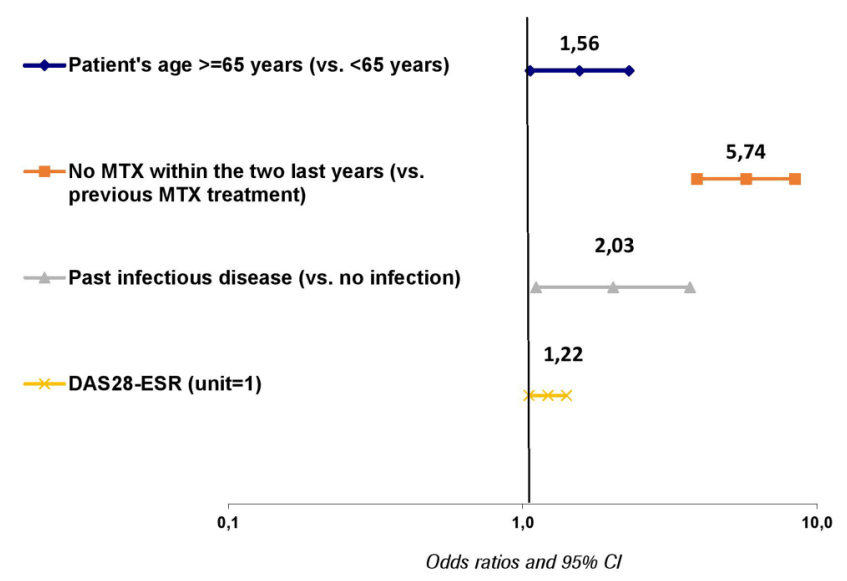

Figure 2 Predictive factors of the use of tocilizumab as monotherapy. MTX, methotrexate; DAS28-ESR, Disease Activity Score 28 joints-Erythrocyte Sedimentation Rate.
Use of cSDMARDs

In the COMBO group, the main combined csDMARD at tocilizumab initiation was MTX $(\mathrm{n}=277,79 \%)$, prescribed at the mean weekly dose of $15.7 \pm 4.5 \mathrm{mg}$ (table 2). Over the study period, 25 combination patients $(31 \%)$ discontinued csDMARDs permanently. In the MONO group, 20 patients $(8.8 \%)$ subsequently received csDMARDs at least once for insufficient response.

\section{Use of oral glucocorticoids}

At the time of their inclusion in the ACT-SOLO study, $66 \%$ of the patients received oral glucocorticoids and they were $50 \%$ in this case at M12 among patients still receiving tocilizumab with no difference between groups. Furthermore, among patients taking glucocorticoids, the proportion of treated patients receiving more than $7.5 \mathrm{mg}$ equivalent prednisone daily decreased during the study from $55 \%$ at inclusion to $40 \%$ at M12 with no difference between groups (table 2).

Using the propensity score, no statistical difference between tocilizumab as MONO and in combination with a csDMARD was observed on oral glucocorticoid prescription at $\mathrm{M} 12 \quad(\mathrm{OR}=0.88 ; 95 \%$ CI 0.56 to 1.39 ;

Table 2 Main treatments of RA over the study period $(\mathrm{N}=577)$

\begin{tabular}{|c|c|c|c|}
\hline & $\begin{array}{l}\text { MONO group } \\
\mathrm{N}=228\end{array}$ & $\begin{array}{l}\text { COMBO group } \\
\mathrm{N}=349\end{array}$ & $\begin{array}{l}\text { All patients } \\
\mathrm{N}=577\end{array}$ \\
\hline \multicolumn{4}{|l|}{ Tocilizumab } \\
\hline Number of infusions & $9.1 \pm 4.3$ & $9.7 \pm 4.0$ & $9.4 \pm 4.1$ \\
\hline Treatment duration (months) & $8.64 \pm 4.30$ & $9.07 \pm 4.00$ & $8.90 \pm 4.12$ \\
\hline Patients with $\geq 1$ modification of tocilizumab treatment, $n(\%)$ & $118(51.8)$ & $153(43.8)$ & $271(47.0)$ \\
\hline Patients with $\geq 1$ change in dose & $35^{\star}(29.7)$ & $45(29.4)$ & $80 \dagger(29.5)$ \\
\hline At least one dose decrease & $29(82.9)$ & $44(97.8)$ & $73(91.3)$ \\
\hline Patients with $\geq 1$ temporary discontinuation & $29(24.6)$ & $50(32.7)$ & $79(29.2)$ \\
\hline Permanent discontinuation & $70(59.3)$ & $93(60.8)$ & $163(60.1)$ \\
\hline \multicolumn{4}{|l|}{$\operatorname{csDMARDs}, n(\%)$} \\
\hline Patients with $\geq 1$ combined csDMARD at inclusion & $0(0.0)$ & 349 (100.0) & $349(60.5)$ \\
\hline MTX & & $268(76.8)$ & \\
\hline Other csDMARDs & & $72(20.6)$ & \\
\hline MTX and other csDMARD & & $9(2.6)$ & \\
\hline Patients with $\geq 1$ combined csDMARD over the study period & $20(8.8)$ & $349(100.0)$ & $369(64.0)$ \\
\hline \multicolumn{4}{|l|}{ Patients with $\geq 1$ change in combined } \\
\hline csDMARDs after inclusion & $12(5.3)$ & $80(22.9)$ & $92(15.9)$ \\
\hline Patients with $\geq 1$ change in dose & $8(3.5)$ & $52(14.9)$ & $60(10.4)$ \\
\hline Patients with $\geq 1$ temporary discontinuation & $1(0.4)$ & $13(3.7)$ & $14(2.4)$ \\
\hline Permanent discontinuation & $4(1.7)$ & $25(7.2)$ & $29(5.0)$ \\
\hline \multicolumn{4}{|l|}{ Oral glucocorticoids, $n(\%)$} \\
\hline \multicolumn{4}{|c|}{ Dose of oral glucocorticoids at M12 (mg/daily equivalent prednisone) } \\
\hline Missing data & $98(43)$ & $142(40.6)$ & $240(41.5)$ \\
\hline 0 & $64(28.1)$ & $103(29.5)$ & $167(28.9)$ \\
\hline$[0-5]$ & $36(15.8)$ & $48(13.75)$ & $84(14.5)$ \\
\hline$[5-7.5]$ & $8(3.5)$ & $23(6.6)$ & $31(5.4)$ \\
\hline$[7.5-10]$ & $13(5.7)$ & $20(5.7)$ & $33(5.7)$ \\
\hline$>10$ & $9(3.9)$ & $13(3.7)$ & $22(3.8)$ \\
\hline
\end{tabular}


$\mathrm{p}=0.581$ ) (data provided in online supplementary material). After univariate analyses, multivariate analysis showed that high glucocorticoid dosage at first tocilizumab infusion $(p<0.0001)$, high patient's global assessment of disease activity $(\mathrm{p}=0.043)$ and overweight were associated to a statistically significant increased risk of glucocorticoids use at M12 ( $\mathrm{p}=0.044)$ (data provided in online supplementary material).

\section{Disease activity}

Considering missing data as failure, the proportion of patients in DAS28-ESR LDA rapidly increased as early as
M3 (from 4\% to 37\% between inclusion and M3 in monotherapy patients; from $7 \%$ to $46 \%$ in combination patients), and then more slowly until M12 (up to $41 \%$ and $44 \%$, respectively) (table 3 ).

Respectively $35 \%$ and $36 \%$ of the MONO and COMBO patients reached DAS28-ESR remission at M12 while these proportions were $2 \%$ and $3 \%$ before the first tocilizumab infusion. The number of missing data, analysed as failure, was large at each follow-up time (148 at M3, 206 at M6 and 253 at M12). Similar favourable evolution of the other efficacy parameters (CDAI, SDAI, ACR20/50/70, EULAR response, HAQ-DI score) was

Table 3 Main efficacy results at M12 (N=577)

$\begin{array}{lll}\text { MONO group } & \text { COMBO group } & \text { All patients } \\ \mathrm{N}=228 & \mathrm{~N}=349 & \mathrm{~N}=577\end{array}$

Disease activity indices and efficacy responses at M12 (missing data not taken into account for analyses)

DAS28-ESR, n/N (\%)

$\operatorname{LDA}(\leq 3.2)$

Remission (<2.6)

CDAI, n/N (\%)

LDA $(\leq 10)$

Remission ( $\leq 2.8)$

SDAI, $\mathrm{n} / \mathrm{N}(\%)$

LDA $(\leq 11)$

Remission ( $\leq 3.3)$

$\mathrm{HAQ}-\mathrm{DI}$ Questionnaire, mean \pm SD

Standard Score

Change between D0 and M12

Disease activity indices and efficacy

responses at M12 (missing data considered as failure)

DAS28-ESR, $\mathrm{n}(\%)$

LDA ( $\leq 3.2)$ at M12

Remission (<2.6) at $\mathrm{M} 12$

CDAI, n/N (\%)

LDA $(\leq 10)$ at M12

Remission $(\leq 2.8)$ at $\mathrm{M} 12$

SDAI, $\mathrm{n} / \mathrm{N}(\%)$

LDA $(\leq 11)$ at M12

Remission ( $\leq 3.3)$ at $\mathrm{M} 12$

ACR20/50/70 responses, $n$ (\%)

ACR20 responders

ACR50 responders

ACR70 responders

EULAR responses, $\mathrm{n}(\%)$

Good or moderate

Patients' quality of life at M12

RAID Score

Score, mean \pm SD

Change between D0 and M12, mean \pm SD

\section{PASS Questionnaire}

Acceptable condition- $\mathrm{n}(\%)$
$94 / 125(75.2)$

$79 / 125$ (63.2)

$71 / 104$ (68.3)

22/104 (21.2)

$70 / 101(69.3)$

23/101 (22.8)

$(n=86)$

$1.06 \pm 0.78$

$(n=74)$

$-0.47 \pm 0.68$

$(n=228)$

$94(41.2)$

79 (34.6)

$71(31.1)$

$22(9.6)$

$70(30.7)$

$23(10.1)$

$74(32.5)$

50 (21.9)

22 (9.6)

$114(50.0)$

( $n=87)$

$3.70 \pm 2.17$

$(n=72)$

$-2.42 \pm 2.29$

$(n=84)$

71 (84.5)
155/199 (77.9)

124/199 (62.3)

$82 / 139$ (59.0)

$30 / 139$ (21.6)

$81 / 136$ (59.6)

$34 / 136$ (25.0)

249/324 (76.9)

203/324 (62.7)

$153 / 243$ (63.0)

$52 / 243$ (21.4)

151/237 (63.7)

$57 / 237$ (24.1)

$(n=132)$

$1.02 \pm 0.69$

( $n=218)$

$1.03 \pm 0.73$

$(n=180)$

$-0.46 \pm 0.65$

$-0.45 \pm 0.62$

$(n=349)$

$(n=577)$

155 (44.4)

124 (35.5)

249 (43.2)

203 (35.2)

82 (23.5)

30 (8.6)

153 (26.5)

52 (9.0)

81 (23.2)

151 (26.2)

57 (9.9)

92 (26.4)

59 (16.9)

166 (28.8)

109 (18.9)

$56(9.7)$

287 (49.7)

$173(49.6)$

(n=134)

( $n=221)$

$3.63 \pm 2.14$

$(n=108)$

$-2.15 \pm 2.34$

$3.66 \pm 2.15$

( $n=180)$

$-2.26 \pm 2.32$

( $n=132)$

( $n=216)$

105 (79.5)

thetic disease-modifying matic drug; COMBO, tocilizumab in combination with csDMARD at inclusion; DAS28, Disease Activity Score 28 joints; ESR, Erythrocyte Sedimentation Rate; EULAR, European League Against Rheumatism; HAQ-DI, health assessment-disease index questionnaire; MONO, tocilizumab as monotherapy at inclusion; PASS, patient acceptable symptom state; RAID, rheumatoid arthritis impact of disease; SDAI, simplified disease activity index. 
observed at M12 (table 3). Considering missing data as failure, numerically more monotherapy patients reached CDAI and SDAI LDA than in COMBO group (respectively, $31 \%$ vs $24 \% ; 31 \%$ vs $23 \%$ ), while remission rates were similar (between $9 \%$ and $10 \%$ ). Very similar results were observed with ACR20 and ACR70 (respectively, $33 \%$ vs $26 \%$; $10 \%$ in both groups). For EULAR response, no differences were observed at each follow-up time between monotherapy and combination patients and $50 \%$ of both groups' patients reached good or moderate EULAR response at M12. As shown on table 3, the mean HAQ-DI score decreased (ie, improved) from the first tocilizumab infusion without differences between patients' groups.

Using the propensity score, no statistical difference between tocilizumab as MONO and in combination with a csDMARD was observed on achievement of DAS28-ESR LDA and remission at M12 (OR=0.95; 95\% CI 0.67 to $1.36 ; \mathrm{p}=0.79$ and $\mathrm{OR}=1.11 ; 95 \%$ CI 0.77 to $1.60, \mathrm{p}=0.57$, respectively) (data provided in online supplementary material). After univariate analyses, multivariate analysis showed that positive rheumatoid factor (RF) or anticitrullinated protein antibodies (ACPA, $\mathrm{p}=0.045$ ) was associated to an increased chance to achieve DAS28-ESR LDA at M12. In contrast, a higher DAS28-ESR at first tocilizumab infusion $(p=0.0004)$ was associated to an increased risk of non-achievement at that time. Using the same analysis method, a higher DAS28-ESR at first tocilizumab infusion $(p<0.0001)$ was associated to a statistically significant increased risk of non-achievement of DAS28-ESR remission at M12 (such as for DAS28-ESR LDA).

\section{Patients' quality of life}

At M12, patients' quality of life improved from the first tocilizumab infusion using RAID score and PASS questionnaire, similarly in MONO and COMBO groups (data provided in online supplementary material).

\section{Safety}

The main safety outcomes of the 603 patients analysed in the safety population are presented in table 4 .

At least one $\mathrm{AE}$ and at least one $\mathrm{SAE}$ were reported in 325 patients $(54 \%)$ and in 74 patients $(12 \%)$, respectively, over the study (median follow-up of patients: 11.4 months; range: 0.0-15.6) and no relevant differences were observed between groups and no new safety signal raised during the study. Among the 463 AEs related to tocilizumab according to investigators, the most frequently reported events $(\geq 5 \%)$ were in the System Organ Classes (SOC) of infections and infestations (171 events reported in 112 patients, 19\%), blood and lymphatic system disorders (98 events reported in 56 patients, $9 \%$ ) and skin and subcutaneous tissue disorders (38 events reported in 29 patients, $<5 \%$ ). Among the 41 related SAEs, the most frequently reported events were in the SOC of infections and infestations (27 events reported in 23 patients, $4 \%$ ). Irrespective of the
SOC, at least one serious and/or medically significant infection was reported in 30 patients $(5 \%)$, including 23 patients $(4 \%)$ with at least one related event (with two patients with related Pneumocystis jiroveci pneumonia). At least one serious and/or medically significant hepatic event was reported in 24 patients $(4 \%)$ without differences between monotherapy $(n=9,3.8 \%)$ and combination $(n=15,4.1 \%)$ patients. One gastrointestinal perforation, three myocardial infarction/acute coronary syndromes and seven malignancies were also reported over the study period. Three deaths were associated with at least one $\mathrm{AE}$, without causal relationship with tocilizumab as assessed by physicians.

\section{DISCUSSION}

At the implementation of the ACT-SOLO study, $40 \%$ of the RA patients received tocilizumab as MONO. Four independent predictive factors of tocilizumab MONOS were shown: at least 65 years of age, no MTX use within the two last years, history of severe infectious disease and higher baseline DAS28-ESR.

If we consider drug retention rate, disease activity and use of steroids at M12 as markers of efficacy, there was no difference between tocilizumab MONO and COMBO groups. Regarding safety, no new signal occurred and no difference was reported between the two groups.

The $40 \%$ relatively high proportion of patients receiving tocilizumab as MONO in this study was explained by intolerance to previous MTX in $74 \%$ of the cases and was higher than findings on biologics from previous studies published until 2011 $17-192224$ in which there was around $30 \%$ of monotherapy patients, with variations according to each bDMARD, rheumatologists' practices in the different countries and times when previous registries were conducted. In more recent non-interventional French studies conducted in tocilizumab-treated patients with RA (inclusions from 2011 to 2012), 38\% of RA patients received monotherapy, ${ }^{25}{ }^{26}$ that is, a close proportion to ACT-SOLO patients. This rate may be explained by the unique position granted to tocilizumab in the EULAR or in the French Society of Rheumatology recommendations when a biological agent had to be used alone. ${ }^{27} 28$

The 577 RA patients included in ACT-SOLO efficacy analysis presented with similar characteristics for age and sex across six non-interventional and four interventional studies conducted in RA patients initiating treatment with tocilizumab after inadequate response to DMARDs. ${ }^{6} 222526$ 29-33 Mean RA duration varied from 7 to 14 years across studies. DAS28 was similar in the non-interventional studies, and higher in the interventional ones, while HAQ-DI score was similar across studies. The proportion of ACT-SOLO patients with previous bDMARDs was similar to the four other noninterventional studies mentioned above.

The less favourable profile of RA monotherapy patients (slightly older and with more comorbidities 
Table 4 Summary of all adverse events over the safety reporting period-safety population $(\mathrm{N}=603)$

\begin{tabular}{|c|c|c|c|}
\hline n (\%) & $\begin{array}{l}\text { MONO group } \\
\mathrm{N}=234\end{array}$ & $\begin{array}{l}\text { COMBO group } \\
\mathrm{N}=369\end{array}$ & $\begin{array}{l}\text { All patients } \\
\mathrm{N}=603\end{array}$ \\
\hline \multicolumn{4}{|l|}{ Adverse events (AEs) } \\
\hline Patients with $\geq 1 \mathrm{AE}$ & $128(54.7)$ & $197(53.4)$ & $325(53.9)$ \\
\hline Number of AEs & 420 & 566 & 986 \\
\hline Patients with $\geq 1 \mathrm{AE}$ related ${ }^{*}$ to tocilizumab & $93(39.7)$ & $129(35.0)$ & $222(36.8)$ \\
\hline Number of AEs related ${ }^{*}$ to tocilizumab & 203 & 261 & 464 \\
\hline Patients with $\geq 1 \mathrm{AE}$ leading to tocilizumab permanent discontinuation & $25(10.7)$ & $35(9.5)$ & $60(10.0)$ \\
\hline Number of AEs leading to tocilizumab permanent discontinuation & 35 & 46 & 81 \\
\hline Patients with $\geq 1 \mathrm{AE}$ leading to death & $2(0.9)$ & $1(0.3)$ & $3(0.5)$ \\
\hline Number of AEs leading to death & 3 & 1 & 4 \\
\hline Patients with $\geq 1 \mathrm{AE}$ related ${ }^{*}$ to tocilizumab leading to death & $1(0.4)$ & 0 & $1(0.2)$ \\
\hline Number of AEs related ${ }^{*}$ to tocilizumab leading to death & 1 & 0 & 1 \\
\hline \multicolumn{4}{|l|}{ Serious adverse events (SAEs) } \\
\hline Patients with $\geq 1 \mathrm{SAE}$ & $31(13.2)$ & $43(11.7)$ & 74 (12.3) \\
\hline Number of SAEs & 51 & 59 & 110 \\
\hline Patients with $\geq 1$ SAE related ${ }^{*}$ to tocilizumab & $12(5.1)$ & $18(4.9)$ & $30(5.0)$ \\
\hline Number of SAEs related ${ }^{*}$ to tocilizumab & 20 & 22 & 42 \\
\hline \multicolumn{4}{|l|}{ Adverse events of special interest (AESIs) $\dagger$} \\
\hline Patients with $\geq 1 \mathrm{AESI}$ & $47(20.1)$ & $73(19.8)$ & $120(19.9)$ \\
\hline Number of AESIs & 75 & 99 & 174 \\
\hline Patients with $\geq 1 \mathrm{AESI}$ related ${ }^{*}$ to tocilizumab & 29 (12.4) & $42(11.4)$ & $71(11.8)$ \\
\hline Number of AESIs related ${ }^{\star}$ to tocilizumab & 43 & 51 & 94 \\
\hline Patients with $\geq 1 \mathrm{AESI}$ leading to tocilizumab permanent discontinuation & $16(6.8)$ & $20(5.4)$ & $36(6.0)$ \\
\hline Number of AESIs leading to tocilizumab permanent discontinuation & 20 & 22 & 42 \\
\hline \multicolumn{4}{|l|}{ Serious adverse events of special interest (SAESIs) } \\
\hline Patients with $\geq 1$ SAESI & $19(8.1)$ & $30(8.1)$ & $49(8.1)$ \\
\hline Number of SAESIs & 26 & 34 & \\
\hline Patients with $\geq 1$ SAESI related ${ }^{*}$ to tocilizumab & $9(3.8)$ & $17(4.6)$ & $26(4.3)$ \\
\hline Number of SAESIs related ${ }^{*}$ to tocilizumab & 16 & 18 & 34 \\
\hline
\end{tabular}

${ }^{*}$ In case of missing information about causal relationship with tocilizumab, the $\mathrm{AE}$ was considered as related to treatment.

†AESIs: anaphylaxis/hypersensitivity reactions, demyelinating disorders, gastrointestinal perforations, malignancies, myocardial infarctions/ acute coronary syndromes, serious and/or medically significant infections, serious and/or medically significant hepatic events, serious and/or medically significant bleeding events and strokes.

csDMARD, conventional synthetic disease-modifying antirheumatic drug; COMBO, tocilizumab in combination with csDMARD at inclusion; MONO, tocilizumab as monotherapy at inclusion.

than combination patients) has been previously reported. ${ }^{18}$ Furthermore, baseline DAS28-ESR was higher in monotherapy patients.

The predictive factors of tocilizumab MONO were consistent findings with those observed in a recent study where bDMARD MONO was preferentially prescribed to older, comorbid patients with longer disease duration, lower BMI, more active disease and more previous bDMARDs. ${ }^{34}$

The median rates of retention in tocilizumab treatment until M12 were not different between patients' groups (in $67 \%$ of monotherapy patients and in $71 \%$ of combination patients). These proportions are lower than previously reported in two observational studies $(82 \%$ and $89 \%),{ }^{31}{ }^{35}$ but similar to the REACTION study $(71 \%)$ and the Danish Biologics (DANBIO) registry (64\%). ${ }^{36} 37$

Complementary analyses focused on the possible effect of tocilizumab use (as MONO or in combination with a csDMARD) on treatment maintenance until M12, prescription of glucocorticoids at M12, achievement of DAS28-ESR LDA and remission at M12. No effect of tocilizumab MONO was shown on these parameters in the ACT-SOLO study. However, in a Swiss registry (3111 patients with several years of follow-up), when all bDMARDs were taken into account for analyses, drug retention was significantly lower in monotherapy $(\mathrm{HR}=1.15, \quad 95 \%$ CI 1.03 to $1.30, \quad \mathrm{p}=0.018) .{ }^{34}$ Furthermore, although findings from the European tocilizumab collaboration of European registries in RA (TOCERA) (with no data from France) showed no difference in tocilizumab MONO retention rate at 1.5 years, $(\mathrm{HR}=1.10,95 \%$ CI 0.87 to $1.39, \mathrm{p}=0.41)$ when compared with combination therapy, a statistically significant difference appeared at 2 years. Tocilizumab retention was indeed shorter in patients under MONO (2.3 years (95\% CI 1.8 to 2.7 ) versus 3.7 years $(95 \%$ CI 3.1 to not estimable) if combination therapies).$^{38}$ Thus, the absence of difference in drug retention rates in the ACT-SOLO study might be related to the duration of follow-up. Dyslipidaemia was surprisingly associated to a statistically significant increased chance of treatment 
maintenance, possibly due to a more cautious management of such patients by physicians, medical teams and patients themselves.

Considering missing data as failure, the proportion of patients in DAS28-ESR LDA increased up to $41 \%$ in the monotherapy group and $44 \%$ in the COMBO group at M12, and $35 \%$ and $36 \%$ of the patients reached DAS28-ESR remission at that time. However, the number of missing data was large, which probably led to underestimation of the improvements observed under treatment. Using the same analysis method, similar results were observed in the French non-interventional SPARE-1 study: $41 \%$ and $33 \%$ of patients in DAS28-ESR LDA and remission at M12, respectively. ${ }^{26}$ By comparison, in the interventional tocilizumab safety and the prevention of structural joint damage (LITHE) study with the same analysis method, ${ }^{9}$ the proportions of patients (treated with tocilizumab 8mg q4w plus MTX) in DAS28-ESR LDA and remission after a 1-year treatment period were higher (64\% and $47 \%$, respectively) when compared to our combination patients data $(44 \%$ and $36 \%)$. After 1-year treatment period, in monotherapy patients included in the phase IVACT-RAY trial, ${ }^{32}$ LDA was $57 \%$ (higher than what was observed in ACT-SOLO monotherapy group, ie $41 \%$ ) and DAS28 remission was similar $(37 \%$ compared with $35 \%$ ). After a 6 -month treatment period, $38 \%$ and $29 \%$ of monotherapy patients were already in DAS28-ESR LDA and remission, respectively, in the ACT-SOLO study, compared with $52 \%$ and $40 \%$ in the phase IV tocilizumab monotherapy versus adalimumab monotherapy for treatment of rheumatoid arthritis (ADACTA) study. ${ }^{33}$ These best results probably reflect the patients' selection as well as the close patient's management in interventional studies.

Excluding missing data from analyses, stronger improvements were observed in the ACT-SOLO in 12-month DAS28-ESR LDA and remission (reached respectively, by $77 \%$ and $63 \%$ of patients) compared with other non-interventional studies (Swedish ARTIS registry: $55 \%$ and $37 \%$; German ROUTINE study: $44 \%$ and 55\%). ${ }^{29}{ }^{30}$ Finally, the ACT-SOLO results are consistent in terms of efficacy on disease activity with all these interventional and non-interventional studies showing a positive control of disease activity observed after 6 months and maintained or slightly improved when studies lasted for 12 months.

The use of tocilizumab was close to the instructions of RoActemra summary of product characteristics (SmPC) (dosing, frequency and time between two infusions) and the reasons leading to tocilizumab MONO were in accordance with the SmPC recommendations: intolerance or contraindications to MTX in most of the cases.

At M12, $50 \%$ of the patients received oral glucocorticoids in both patient groups compared with $66 \%$ at baseline. A glucocorticoids sparing effect was seen in both MONO and combination patients. There were $74 \%$ of them taking $\leq 5 \mathrm{mg}$ /day of equivalent prednisone at M12 (ie, the recommended maximal acceptable daily dose ${ }^{39}$ ), $79 \%$ in
MONO group and $73 \%$ in COMBO group, compared with $53 \%$ (both groups) at first tocilizumab infusion.

\section{Safety results}

The safety outcomes $(37 \%$ with at least one AE related to tocilizumab according to physicians) confirmed previous findings about tocilizumab, as observed in the French non-interventional SPARE-1 study (41\% of patients in this case).$^{26}$ In particular, a slow incidence of serious and/or medically significant infections (at least one in 30 patients, 5\%) was reported in the ACT-SOLO study as observed in the French registries of RA-treated patients (auto-immunité et rituximab (AIR), orencia and rheumatoid arthritis (ORA) and registry - roactemra (REGATE): 55 events, ie, 5.2 serious infections/ 100 patient-years).$^{40}$ However, adverse events were under-reported in these non-interventional studies compared with the TOWARD and ACT-SURE clinical trials $(73 \%$ and $77 \%$ of patients with at least one $\mathrm{AE}$ after 24 weeks of treatment, respectively). ${ }^{6} \quad 41$ Laboratory values were not systematically assessed during the study, and only reported when linked to an adverse event. This might explain the absence of difference between monotherapy and combination patients in reported hepatic events in contrast of what was shown in the ACT-RAY study. ${ }^{32}$

Thus, despite the less favourable profile of monotherapy patients, there was no difference in terms of frequency, nature and severity between the two groups.

That being said, no new findings were shown in the ACT-SOLO study by comparison with the tocilizumab SmPC and with pivotal studies.

The strength and limitations are those of observational studies.

To remedy the large number of missing data in the ACT-SOLO study, numbers usually observed in noninterventional studies, sensitivity analyses were carried out. They confirmed initial findings regarding predictive factors of tocilizumab MONO and efficacy parameters.

\section{CONCLUSION}

This non-interventional 608-patient study conducted in RA patients confirms in real-life conditions that more than one-third of them $(40 \%)$ started tocilizumab treatment as MONO. This therapeutic strategy was associated with no MTX treatment within the past 2 years, elderly patients (at least 65 years old), history of severe infection and more active disease. Twelve months after the first tocilizumab infusion, clinical results showed no differences between patients receiving the drug as monotherapy or in combination with a csDMARD. The use of tocilizumab was in accordance with treatment SmPC in most of the patients. No new safety signal arose during the study.

\section{Author affiliations}

${ }^{1}$ Department of Rheumatology, University Hospital, Lille, France

${ }^{2}$ Department of Rheumatology, University Hospital, Dijon, France

${ }^{3}$ Department of Rheumatology and Internal Medicine, Croix Saint Simon Hospital, Paris, France 
${ }^{4}$ Medical Department, Chugai Pharma, Paris, France

${ }^{5}$ Department of Clinical Operations, Roche, Boulogne-Billancourt, France

${ }^{6}$ Department of Rheumatology, Lyon Sud hospital, Lyon, France

Acknowledgements The authors thank all the patients and physicians who participated in the ACT-SOLO study. For this study, support was provided by ASCOPHARM Groupe NOVASCO (monitoring and logistic services),

QUINTILES (safety management), LINCOLN (data management), STATITEC (statistical analyses), and AUXESIA (manuscript preparation).

Contributors All authors contributed to the conception or design of the work, data analysis and interpretation, critical revision of the article and final approval of the version to be published. R-MF, J-FM and JT were involved in the study design and participated in the interpretation of study results. R-MF, J-FM, JT and PC were investigators, involved in the study data collection and in the critical revision and approval of the final version of the manuscript.

Funding This study was funded by Roche SAS and Chugai Pharma France. Roche SAS and Chugai Pharma France were involved in the study design, data collection, data analysis and preparation of the manuscript.

Competing interests R-MF, J-FM and JT were the scientific committee of the study. R-MF is member of the medical board and consultant for Roche Chugai France.

Ethics approval The study was approved by Comité Consultatif sur le Traitement de l'Information en Matière de Recherche dans le Domaine de la Santé (Consultative Committee on Information Processing for Research in the Field of Health) and was validated by the Commission Nationale de I'Informatique et des Libertés (Independent Administrative Authority Protecting Privacy and Personal Data).

Provenance and peer review Not commissioned; externally peer reviewed.

Data sharing statement Clinical Study Report may be requested by email. at idier@chugai-pharm.fr

Open Access This is an Open Access article distributed in accordance with the Creative Commons Attribution Non Commercial (CC BY-NC 4.0) license, which permits others to distribute, remix, adapt, build upon this work noncommercially, and license their derivative works on different terms, provided the original work is properly cited and the use is non-commercial. See: http:// creativecommons.org/licenses/by-nc/4.0/

\section{REFERENCES}

1. Guillemin F, Saraux A, Guggenbuhl P, et al. Prevalence of rheumatoid arthritis in France: 2001. Ann Rheum Dis 2005;64:1427-30.

2. Lee DM, Weinblatt ME. Rheumatoid arthritis. Lancet 2001;358:903-11.

3. Scott DL. Radiological progression in established rheumatoid arthritis. J Rheumatol Suppl 2004;69:55-65.

4. Welsing PM, van Gestel AM, Swinkels $\mathrm{HL}$, et al. The relationship between disease activity, joint destruction, and functional capacity over the course of rheumatoid arthritis. Arthritis Rheum 2001;44:2009-17.

5. Emery P, Keystone E, Tony HP, et al. IL-6 receptor inhibition with tocilizumab improves treatment outcomes in patients with rheumatoid arthritis refractory to anti-tumour necrosis factor biologicals: results from a 24-week multicentre randomised placebo-controlled trial. Ann Rheum Dis 2008;67:1516-23.

6. Genovese MC, McKay JD, Nasonov EL, et al. Interleukin-6 receptor inhibition with tocilizumab reduces disease activity in rheumatoid arthritis with inadequate response to disease-modifying antirheumatic drugs: the tocilizumab in combination with traditional disease-modifying antirheumatic drug therapy study. Arthritis Rheum 2008;58:2968-80.

7. Smolen JS, Beaulieu A, Rubbert-Roth A, et al. Effect of intreleukin-6 receptor inhibition with tocilizumab in patients with rheumatoid arthritis (OPTION study): a double-blind, placebo controlled randomised trial. Lancet 2008;371:987-97.

8. Jones $\mathrm{G}$, Sebba A, Gu J, et al. Comparison of tocilizumab monotherapy versus methotrexate monotherapy in patients with moderate to severe rheumatoid arthritis: the AMBITION study. Ann Rheum Dis 2010;69:88-96.
9. Kremer J, Le Loët X, Halland AM, et al. Safety of tocilizumab (TCZ) in combination with methotrexate (MTX): 2-year data from LITHE. Ann Rheum Dis 2010;69(Suppl 3):387

10. Nishimoto N, Hashimoto J, Miyasaka N, et al. Study of active controlled monotherapy used for rheumatoid arthritis, an IL-6 inhibitor (SAMURAI): evidence of clinical and radiographic benefit from an $\mathrm{x}$ ray reader-blinded randomised controlled trial of tocilizumab. Ann Rheum Dis 2007;66:1162-7.

11. Nishimoto N, Miyasaka N, Yamamoto K, et al. Study of active controlled tocilizumab monotherapy for rheumatoid arthritis patients with an inadequate response to methotrexate (SATORI): significant reduction in disease activity and serum vascular endothelial growth factor by IL-6 receptor inhibition therapy. Mod Rheumatol 2009;19:12-19.

12. DeWitt EM, Lin L, Glick HA, et al. Pattern and predictors of the initiation of biologicagents for the treatment of rheumatoid arthritis in the United States: an analysis using a large observational data bank. Clin Ther 2009;31:1871-80.

13. Smolen JS, Aletaha D, Bijlsma JW, et al. Treating rheumatoid arthritis to target: recommendations of an international task force. Ann Rheum Dis 2010;69:631-7.

14. Smolen JS, Landewé R, Breedveld FC, et al. EULAR recommendations for the management of rheumatoid arthritis with synthetic and biological disease-modifying antirheumatic drugs. Ann Rheum Dis 2010;69:964-75.

15. HAS. Polyarthrite rhumatoïde évolutives graves. Guide-Affections de longue durée. Avril 2008. http://www.has-sante.fr/ (accessed 22 Mar 2016)

16. Hetland ML, Christensen IJ, Tarp U, et al. Direct comparison of treatment responses, remission rates, and drug adherence in patients with rheumatoid arthritis treated with adalimumab, etanercept, or infliximab: results from eight years of surveillance of clinical practice in the nationwide Danish DANBIO registry. Arthritis Rheum 2010;62:22-32.

17. Mariette $X$, Gottenberg JE, Ravaud $P$, et al. Registries in rheumatoid arthritis and autoimmune diseases: data from the French registries. Rheumatology (Oxford) 2011;50:222-9.

18. Soliman MM, Ashcroft DM, Watson KD, et al. Impact of concomitant use of DMARDs on the persistence with anti-TNF therapies in patients with rheumatoid arthritis: results from the British Society for Rheumatology Biologics Register. Ann Rheum Dis 2011;70:583-9.

19. Listing J, Strangfeld A, Rau R, et al. Clinical and functional remission: even though biologics are superior to conventional DMARDs overall success rates remain low-results from RABBIT, the German biologics register. Arthritis Res Ther 2006;8:R66.

20. Fries JF, Spitz P, Kraines RG, et al. Measurement of patient outcome in arthritis. Arthritis Rheum 1980;23:137-45.

21. Gossec L, Dougados M, Rincheval N, et al. The elaboration of the preliminary Rheumatoid Arthritis Impact of Disease (RAID) score: a EULAR initiative. Ann Rheum Dis 2009;68:1680-5.

22. Gossec L. Fatigue in patients with rheumatoid arthritis treated with tocilizumab (Actemra®) in real life: clinically relevant improvement in $62 \%$ of patients at 4 months and rapid onset of action. The PEPS study. Clin Exp Rheumatol 2015;33:664-70.

23. Morel J, Duzanski MO, Bardin T, et al. Prospective follow-up of tocilizumab treatment in 764 patients with refractory rheumatoid arthritis: Tolerance and efficacy data from the French registry Regate (REGistry -RoAcTEmra). Arthritis Rheum 2012;64(Suppl 10):351.

24. Pers $\mathrm{YM}$, Fortunet $\mathrm{C}$, Constant $\mathrm{E}$, et al. Predictors of response and remission in a large cohort of rheumatoid arthritis patients treated with tocilizumab in clinical practice. Rheumatology (Oxford) 2014;53:76-84.

25. Morel J, Duzanski MO, Cantagrel A, et al. Prospective follow-up of tocilizumab treatment in 1100 patients with refractory rheumatoid arthritis: tolerance data from the French registry REGATE (REGistry -RoAcTEmr). Ann Rheum Dis 2013;72(Suppl 3):456.

26. Saraux A, Rouanet S, Flipo RM, et al. Glucocorticoid-sparing in patients suffering from rheumatoid arthritis and treated with tocilizumab: the SPARE-1 study. Clin Exp Rhumatol 2016;34:303-10.

27. Smolen JS, Landewé R, Breedveld FC, et al. EULAR recommendations for the management of rheumatoid arthritis with antirheumatic drugs synthetic and biological disease-modifying: 2013 update. Ann Rheum Dis 2014;73:492-509.

28. Gaujoux-Viala C, Gossec L, Cantagrel A, et al. Recommendations of the French Society for Rheumatology for managing rheumatoid arthritis. Joint Bone Spine 2014;81:287-97.

29. Forsblad-d'Elia H, Bengtsson K, Kristensen LE, et al. Drug Survival, Efficacy and Predictors for survival on tocilizumab in real-life patients with rheumatoid arthritis; results from the Swedish Biologics Register. Arhtritis Rheum 2012;64(Suppl):S201. 
30. Burmester GR, von Hinüber U, Richter $\mathrm{C}$, et al. Tocilizumab administered in daily clinical practice-Final results of the German non-interventional study "ROUTINE". Ann Rheum Dis 2013; 72:A452.

31. Balsa A, Tovar Beltrán JV, Cáliz R, et al. Patterns of use and dosing of tocilizumab in the treatment of patients with rheumatoid arthritis in routine clinical practice: the ACT-LIFE study. Rheumatol Int 2015;35:1525-34

32. Dougados M, Kissel K, Conaghan PG, et al. Clinical, radiographic and immunogenic effects after 1 year of tocilizumab-based treatment strategies in rheumatoid arthritis: the ACT-RAY study. Ann Rheum Dis 2014;73:803-9.

33. Gabay C, Emery $\mathrm{P}$, van Vollenhoven $\mathrm{R}$, et al. Tocilizumab monotherapy versus adalimumab monotherapy for treatment of rheumatoid arthritis (ADACTA): a randomised, double-blind, controlled phase 4 trial. Lancet 2013;381:1541-50.

34. Gabay C, Riek M, Scherer A, et al. SCQM collaborating physicians. Effectiveness of biologic DMARDs in monotherapy versus in combination with synthetic DMARDs in rheumatoid arthritis: data from the Swiss Clinical Quality Management Registry. Rheumatology (Oxford) 2015;54:1664-72.

35. Hishitani $Y$, Ogata A, Shima $Y$, et al. Retention of tocilizumab and anti-tumour necrosis factor drugs in the treatment of rheumatoid arthritis. Scand J Rheumatol 2013;42:253-9.
36. Leffers HC, Ostergaard M, Glintborg B, et al. Efficacy of abatacept and tocilizumab in patients with rheumatoid arthritis treated in clinical practice: results from the nationwide Danish DANBIO registry. Ann Rheum Dis 2011;70:1216-22.

37. Takeuchi T, Tanaka Y, Amano K, et al. Clinical, radiographic and functional effectiveness of tocilizumab for rheumatoid arthritis patients-REACTION 52-week study. Rheumatology (Oxford) 2011;50:1908-15.

38. Gabay C, Riek M, Hetland M, et al. Effectiveness of tocilizumab with and without synthetic disease-modifying antirheumatic drugs in rheumatoid arthritis: results from a European collaborative study. Ann Rheum Dis 2016;75:1336-42.

39. Ruyssen-Witrand A, Mouterde G, Dernis E, et al. Tolerance of glucocorticoids in rheumatoid arthritis: recommendations for clinical practice based on data from the literature and experts opinion. Revue du Rhumatisme 2008;75:S20-6.

40. Gottenberg JE, Morel J, Ravaud P, et al. Tolerance of rituximab, abatacept and toocilizumab in common practice: analysis of the 3 registries of the French Society of Rheumatology (AIR, ORA and REGATE). Ann Rheum Dis 2015;74:78-9.

41. Bykerk V, Ostör A, Alvaro-Gracia J, et al. Tocilizumab treatment in patients with rheumatoid arthritis and an inadequate response to DMARDs and/or TNF inhibitor therapy: ACT SURE preliminary results. Ann Rheum Dis 2010;69(Suppl 3):379. 